\title{
Numerous Stones of the Common Bile Duct
}

Key words: numerous, stones, common bile duct, endoscopic biliary drainage

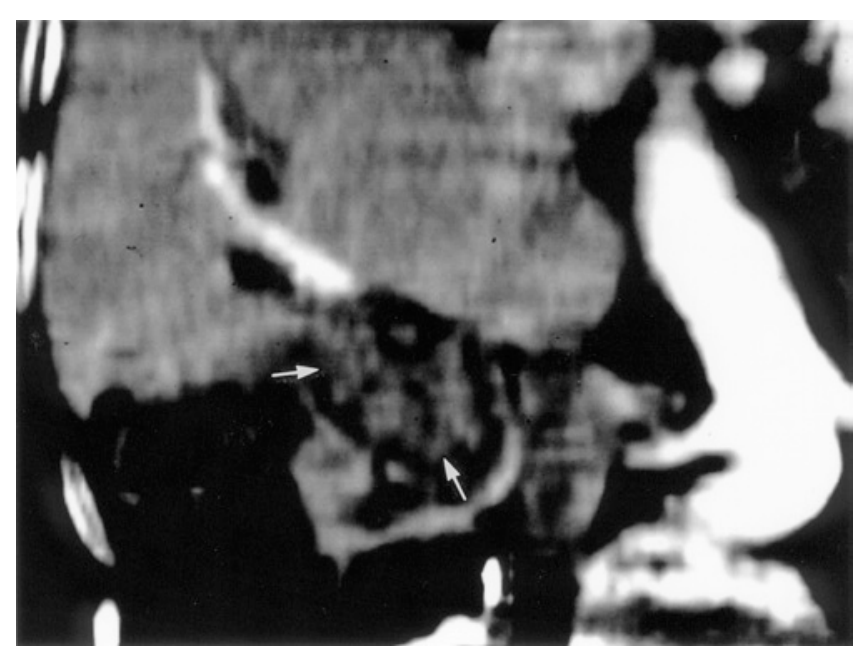

Figure 1. The computed tomography scan of the abdomen showed multiple stones (arrows) in the dilated common bile duct and the dilated intrahepatic bile ducts.

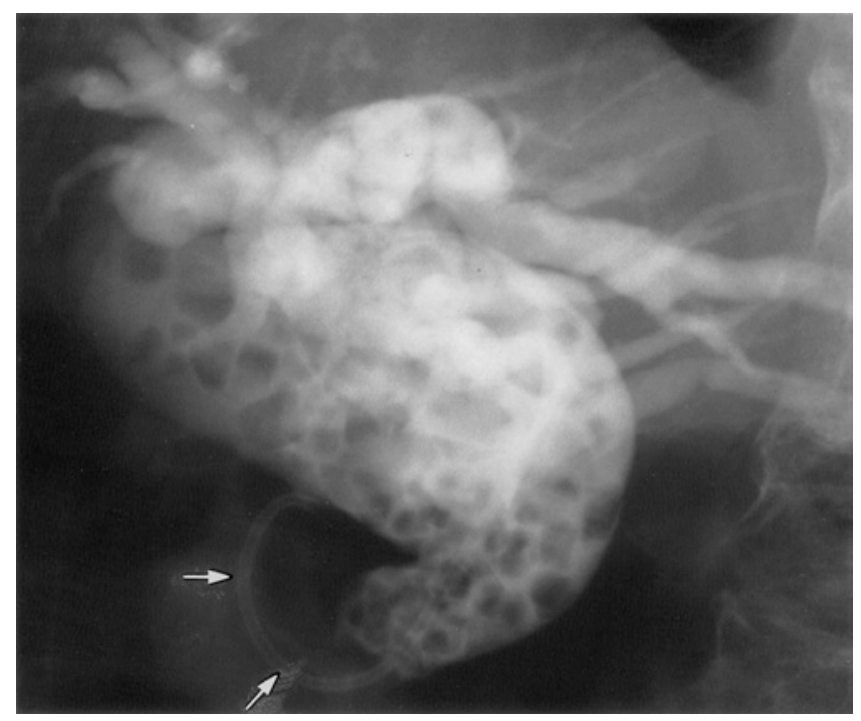

Figure 2. The endoscopic retrograde cholangiography demonstrated numerous stones in the dilated common bile duct. The drainage tube stent (arrows) was inserted into the common bile duct. The gallbladder was not visualized.

A 84-year-old woman who underwent a displacement of ascending aorta with dissecting aortic aneurysm two weeks previously visited our outpatient clinic because of epigastralgia. She had undergone a cholecystectomy twelve years earlier. The abdominal ultrasonography and computed tomography scan revealed multiple calculi (arrows) in the dilated common bile duct (CBD) and the dilated intrahepatic bile ducts (IHBD) (Fig. 1). The endoscopic retrograde cholangiography showed numerous filling defects, less than $10 \mathrm{~mm}$, in the dilated CBD and the dilated IHBD. An anomalous junction of pancreaticobiliary duct was not found. We selected not surgery but endoscopic biliary drainage (tube stent) (arrows) because of the other disease complications (Fig. 2).

Akihiro OKano, Hiroshi Takakuwa and Yuji Matsubayashi

From the Department of Gastroenterology, Tenri Hospital, Tenri

Received for publication February 15, 2005; Accepted for publication April 16, 2005

Reprint requests should be addressed to Dr. Akihiro Okano, the Department of Gastroenterology, Tenri Hospital, 200 Mishima-cho, Tenri, Nara 632-8552 